Manuscript received December 13, 2019; accepted for publication July 1, 2020; published online February 12, 2021. Issue published May 1, 2021.

1 Department of Civil Engineering, University of Alicante, Carretera San Vicente del Raspeig, $\mathrm{s} / \mathrm{n}$, 03690 San Vicente del Raspeig, Alicante, Spain (Corresponding author), e-mail: belen.ferrer@ua.es, (1) https://orcid.org/0000-00028455-5534

2 University of Alicante, Carretera San Vicente del Raspeig, $\mathrm{s} / \mathrm{n}$, 03690 San Vicente del Raspeig, Alicante, Spain

${ }^{3}$ Institute of Physics Applied to Sciences and Technologies, University of Alicante, Carretera San Vicente del Raspeig, $s / \mathrm{n}$, 03690 San Vicente del Raspeig, Alicante, Spain, (1) https://orcid. org/0000-0002-9206-4591
B. Ferrer, ${ }^{1}$ C. Nostas, ${ }^{2}$ and D. Mas $^{3}$

\section{Evaluation of a Simple and Affordable Image-Based Procedure to Measure Particle Size Distribution}

\section{Reference}

B. Ferrer, C. Nostas, and D. Mas, "Evaluation of a Simple and Affordable Image-Based Procedure to Measure Particle Size Distribution," Geotechnical Testing Journal 44, no. 3 (May/ June 2021): 608-621. https://doi.org/10.1520/GTJ20190457

\section{ABSTRACT}

The classical measurement method of particle size for sands and gravels is sieving, but in recent years, imaging methods have been shown to be more reliable. However, imaging procedures require specialized devices (such as Camsizer), proprietary software (such as Optgran-CS), dedicated illumination, or other issues. Furthermore, image resolution in digital cameras improves constantly over time, including in cameras in cellular phones, which leads to the idea that using a standard commercial device and a simple procedure could give results that could be accurate enough. In this work, we first define a simple way of finding the particle size distribution without using any special device, only with a commercial camera and a wellknown free image-processing software. Once defined, we analyze the error magnitude and dispersion of the method with different samples and light intensities. To make the procedure even more accessible, the use of a smartphone camera is also studied. The results show that the method can be used taking into account a correction factor within a narrow interval, which makes this simple method also robust.

\section{Keywords}

image processing, particle size distribution, watershed segmentation, Feret diameters

\section{Introduction}

The knowledge of the size and shape of particles that form a body is required as part of a classification task in such very different science branches as biology (Marinelli et al. 1990), medicine (Corvari et al. 2015), geology (Xiao, Lee, and Wang 2002), agronomy (Li et al. 2008), mining (Annegarn et al. 1987), or industry (Motte et al. 2017), among others. In civil engineering, these characteristics are the basis for the study of soils, base 
and subbase of roads, concrete, asphalt pavements, or gravity dams, among many others. Once the particle size is known, the granulometric distribution can be obtained, which is the key to classifying the soil and therefore obtaining a first approximation of some important soil properties, such as permeability (Hazen 1892, 1911), strength (Cavarretta, Coop, and O'Sullivan 2010), or compressibility (Kodikara, Islam, and Sounthararajah 2018).

The most common method to measure the particle size is mechanical sieving, which consists of measuring the quantity that passes (or is retained) by a sieve with a particular opening (UNE-EN 933-1:2012, Tests for Geometrical Properties of Aggregates - Part 1: Determination of Particle Size Distribution - Sieving Method). Despite being the most used method, sieving has some drawbacks in the determination of particle size and shape: the opening is squared, and the length of its side is used as the size of the particle that can pass through the opening (Fernlund, Zimmerman, and Kragic 2007). However, if the particle has an elongated shape, there is great uncertainty in the measurement, as the particle can pass or be retained depending on the relative alignment between its longest side and the sieve opening shape. Additionally, good sieve results depend on the expertise of the operator (Abbireddy and Clayton 2009).

In any case, as a tridimensional body, the size of a particle cannot be defined by one single length. There are other techniques, such as single particle optical sizing, laser diffraction, electric sensing zone sedimentation, or imaging. Among them, imaging is the technique that is applicable to a larger range of particle sizes (between $0.5 \mu \mathrm{m}$ and $100 \mathrm{~mm}$; Abbireddy and Clayton 2009). Also, with these techniques, two main dimensions of the particle are available for measurement, thus revealing more information than the other techniques, which is one of the reasons that these techniques have been noticeably growing in popularity recently.

Digital image analysis techniques have been used in the literature to determine the particle size: Chávez et al. (2015) developed a method to find the granulometry in sedimentary deposits based on color image segmentation; Sarocchi, Borselli, and Macías (2005) used image methods to find granulometric profiles of pyroclastic deposits; Butler, Lane, and Chandler (2001), Graham, Reid, and Rice (2005), and Buscombe, Rubin, and Warrick (2010) used methods related to digital image analysis to find the granulometry of fluvial sediments on a surface. The complete granulometric distribution of a particle group can be found by image processing, as Raschke and Hryciw have shown through a semiautomatic method for granular soils with particles between 0.023 and $5.7 \mathrm{~mm}$ (Raschke and Hryciw 1997). They also compared the results given by image methods with those obtained by sieving, finding some deviations of the granulometric curves. This comparison was also done by Mora, Kwan, and Chan (1998) for coarse particles. In that paper, particles were carefully spread in the tray without contact between them, and the particle size was determined by direct pixel count for each particle, without any previous image processing. That leads to finding larger particle sizes in the image than sieving by a factor of 10-20\%. Thresholding and watershed were used by Ghalib and Hryciw (1999) to individually identify each particle without isolating them manually, but in this paper, manual pixel count was still used to measure the size of each particle. They also analyzed the correction factors differentiating among elongated, subelongated, subrounded, and rounded particles, ranging from 1.44 for elongated particles to 1 for rounded ones. More recently, Kumara, Hayano, and Ogiwara (2012), Altuhafi, O’Sullivan, and Cavarretta (2013), and Dipova (2017) also used thresholding and watershed to study the size distribution in gravel and sand. The influence of different light intensities was not analyzed in any of these cases, nor was the possibility of using simple setups that may allow in situ analysis.

One problem that arises with the use of images to determine the particle size is the change from mass to area to compare the results given by images (related with particle area) with those given by sieving (related with particle mass). To solve this, some conversion methods have been studied. Mora, Kwan, and Chan (1998) demonstrate that thickness (dimension perpendicular to the image) and density are not relevant to convert image results into sieve results. However, using that conversion, the curve they obtained through image analysis always shows higher sizes than those obtained by mechanical sieving. According to Mora, Kwan, and Chan (1998), this effect is due to the squared shape of the sieve aperture, which prevents the pass of particles that exactly fit to its diagonal because of the limitations of the squared angle. 
Using images to measure particle size also has the advantage of giving information about the particle shape. When a particle is lying on a surface, the smallest dimension is always perpendicular to the surface; therefore, we can obtain information from an image about the dimensions and shape of the largest and middle sizes. Using that information, new shape parameters can be defined based on the particle morphology, such as the figure that can be inscribed or circumscribed on the particle and its relation to the particle area. Parameters to measure flatness and sphericity were defined by Barksdale et al. (1991), while parameters to measure elongation, planarity, shape, and roughness were defined by Kuo, Rollings, and Lynch (1998), and convexity and fullness ratio were defined by Mora and Kwan (2000). With this deep knowledge of the particle shape, the influence of that shape in mechanical sieving was analyzed by Fernlund (1998) and Kwan, Mora, and Chan (1999).

One important issue of the image-based methods is the contrast between particle and background and the isolation of particles in the image. The problem of contrast has commonly been solved by some lamps added to the lateral parts of the camera (Mora, Kwan, and Chan 1998) or, with more complexity, using a glass tray and illuminating it from below (Dipova 2017). Regarding the isolation of particles, most researchers do it manually during sample preparation to take the images (Mora, Kwan, and Chan 1998). This leads to the problem of needing a larger tray for the same quantity of sample, which means a poorer image resolution. In Dipova (2017), this is solved by using a continuous track tray that moves under the camera and taking periodical shots to have all of the sample recorded.

In this work, we first describe a method to obtain particle granulometry on granular soils and shape parameters using a free software such as ImageJ and a commercial camera, with no additional elaborative or expensive devices set up but a camera and a tray. The final aim is to define a fast, cheap and simple procedure to obtain the particle granulometry. The influence of light intensity on the results is also studied by using four different intensities for each image. The procedure is applied on six different samples of granular soils, and the results are compared with those obtained through mechanical sieving. To make the procedure even more affordable, we also propose to use a cell phone as a digital camera. That would provide the technique to nonspecialized soil laboratory people, such as technical on-site staff, students, or almost anyone interested, and permits making a fast in situ soil characterization with only a cell phone, a laptop, and a planar surface that provides a uniform background.

\section{Materials and Methods}

\section{MATERIALS}

Six different granular soil samples obtained from Alicante (Spain) were used. All samples are mixes of gravel and sands with a range size from 0.06 to $20 \mathrm{~mm}$; the particles of 4 samples (named M1, M2, M5, and M6) were more or less rounded, while the other two samples (M3 and M4) had sharp particles. The size of the samples used for imaging was larger than $500 \mathrm{~g}$ in all cases. For the imaging experiment, two different types of camera were used: a Nikon D5100 and the camera of an iPhone 6s Plus, with resolutions of 16.2 and 12 MP, respectively. The obtained ratio $\mathrm{px} / \mathrm{mm}$ slightly varies between samples since the camera lens was slightly adjusted between consecutive experiments, but in general, it is between 8.1 and 9.3. Metallic trays were used to contain the samples and take the images; black cardboard was used at the bottom of the tray to eliminate direct light reflection and increase the contrast between particles and the background. To avoid shadows, 2 small light-emitting diode (LED) panels with 9.6 W each and color temperatures ranging from 3,200 to 5,600 K were used. Both panels were equipped with a diffuser filter and a small tripod to allow its stable vertical position on a table. To analyze the images, the free software ImageJ was used.

\section{EXPERIMENT PROCEDURE}

For all soil samples, two experiments were done. The first one is a mechanical sieving following the procedure given in UNE-EN 933-1:2012. Before sieving, the samples were homogeneously divided and dried until constant weight. 
The second experiment is related to the image acquisition. Because of the whitish color of all samples, black cardboard was set at the bottom of a metallic tray to increase the contrast against the background. The sample was then put in the tray on the cardboard. Regarding this operation, some clarifications must be done. The material is laid out and spread in one layer but not with special care to avoid particle contact. This means that some particles will touch each other but all of them will be in contact with the tray, not allowing overlapping between particles. This is an easy and quick procedure that can be done just by using one hand and the appropriate relation between the size of the tray and the quantity of sample used. Two LED lamps were located on both sides of the tray so that illumination was perpendicular to the table. The light was adjusted to four different intensity levels for each image. Finally, a distance reference (a ruler) was put on the bottom of the tray to have an in-image reference for the pixel to millimeter conversion (fig. 1).

To capture the image, a remote trigger was used with the Nikon camera to avoid vibrations caused by camera manipulation. When using the iPhone camera, it was held by hand without any tripod and the picture was taken manually; only one light intensity was used for this camera. In figure 2, a summary of all images taken for all samples is shown.

\section{IMAGE ANALYSIS}

All image analysis was done using the free software ImageJ. The first step is to find a relation between image and real dimensions; to do that, the "Set scale" option is used together with the ruler that appears in each image. After doing that, a px/mm ratio between 8.1 and 9.3 was obtained for all images. Secondly, a region of interest is selected by taking only the surface with particles in the image. Then, as all images are in RGB mode, the three-color channels are split and the one with the highest contrast is selected for further processing. In all the tests performed here, the red channel had the best contrast and therefore it was selected.

The following step is to binarize the image. To do that, the "Threshold" tool is used with the goal of having the particle in black and the background in white. The value selected as threshold has a key importance, as it can affect the size of the particles in the binarized image. To find the correct threshold for the binarization, the histogram was used. For this kind of images, the histogram is always bimodal, with a minimum value that splits the intensities into two groups: those belonging to the particle and those belonging to the background (fig. 3). Although the threshold level can be selected manually, we propose using Otsu's method (Wikipedia 2020), which separates the pixels into two classes, foreground and background, by minimizing the intensity variance in each of the groups (Sezgin and Sankur 2004).

Once binarized, the next step consists of isolating the different particles. This task is needed because we allowed the particles to touch in the sample preparation to take the images. To isolate the particles, a watershed

\section{FIG. 1}

Setup for the image acquisition with Nikon D5100.

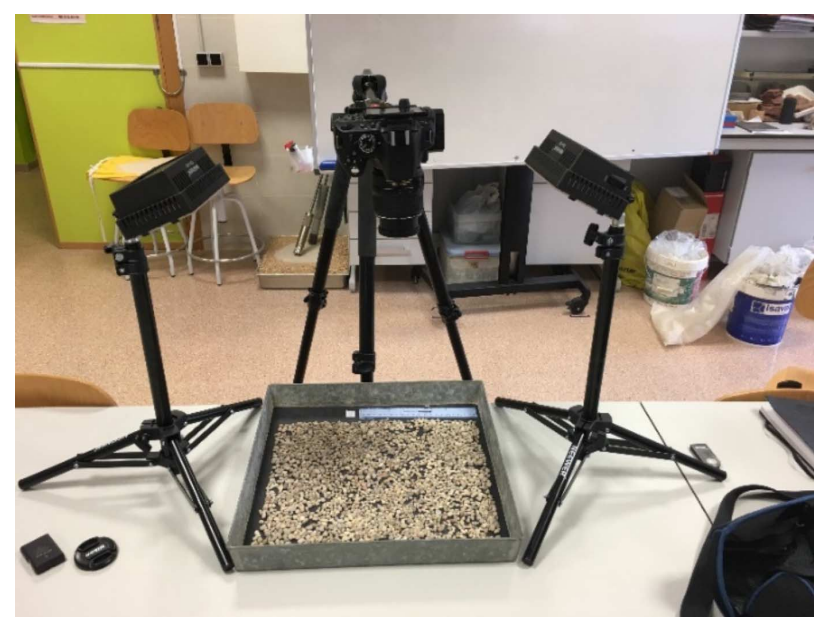


FIG. 2 Summary of images taken for the six samples with different intensity lights and devices. The arrangement of particles in the trays can be also seen in this image.

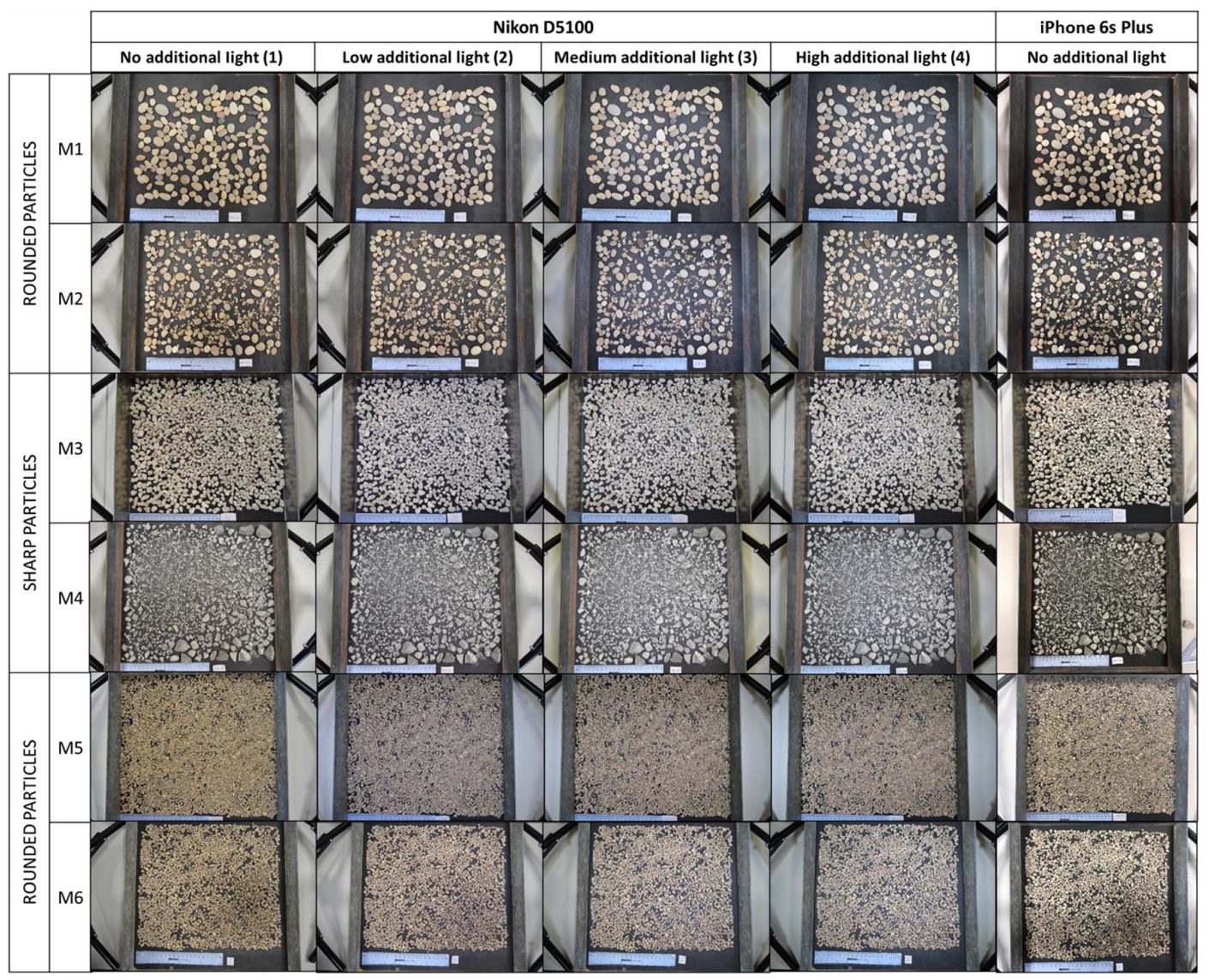

FIG. 3

Typical threshold

for a granular image

with a homogeneous

background.

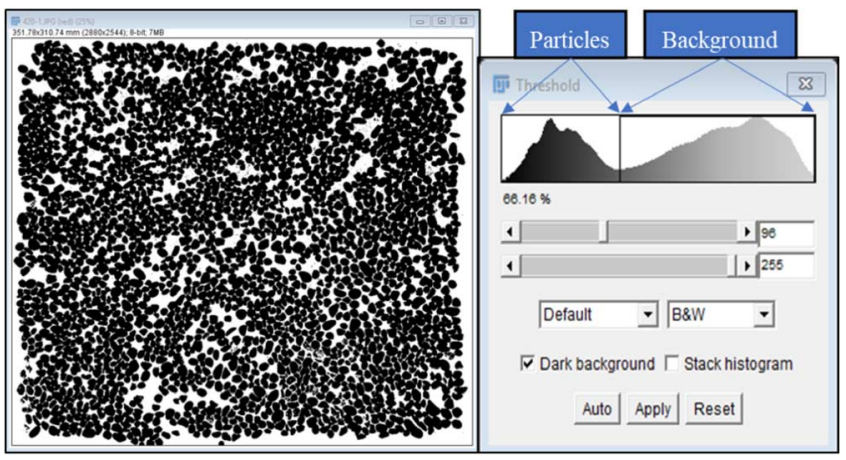

operation was used. The watershed procedure is fully described in Gonzalez and Woods (2008) and briefly consists of calculating the distance from every pixel belonging to an object to its nearest border. Then, a "water source" is located in each local minimum, the region is flooded up to a level where different water sources meet, and a barrier between the regions is set at that point. 
One of the reported problems of the watershed procedure is that local concavities in the object region may produce a false inner boundary, leading to an oversegmentation and therefore to a smaller granulometry than reality (fig. 4). Fortunately, after a visual inspection, we noticed that this happens only in a few cases, and the cost of refining the algorithm in computing time and complexity caused us to assume the error and analyze its influence in the final results. We will come back to this issue in the "Results" section.

Once all particles are isolated, the following step is to identify and characterize them. To do that, the command "Analyze particles" in ImageJ can be used, obtaining the number of objects and their size. Among the output parameters that can be selected, we chose area and Feret diameters (Dipova 2017). Those values, which represent the distance between two parallel lines that are tangent to the particle boundary, have already been used by other researchers to analyze the size and shape of particles through image analysis (Fernlund 1998). In particular, the maximum and minimum Feret distances give a good approximation to the largest and medium particle sizes-let us recall that the minimum size is always perpendicular to the tray and therefore cannot be measured by image analysis. The whole image processing is summarized in figure 5 .

\section{GRANULOMETRY AND ERRORS}

The results obtained through image analysis are the values of area and maximum and minimum Feret diameters for each particle. The following step, outside ImageJ, is to make some simple calculations to obtain the granulometry. To that end, we have calculated the equivalent particle dimension given by Dipova (2017) that takes into account the squared shape of the sieve opening, where $F_{\min }$ is the minimum Feret diameter:

$$
D_{e}=\frac{F_{\min }}{\sqrt{2}}
$$

All the equivalent dimensions were then arranged in decreasing order, and the equivalent retained percentage was determined using equation (2), which can be easily deduced from equation (3) in Mora, Kwan, and Chan (1998):

\section{FIG. 4}

Example of

oversegmentation done in some particles. Real image is shown in the right and result of segmentation is shown in the left. Colored ellipses show some particles that were oversegmented.

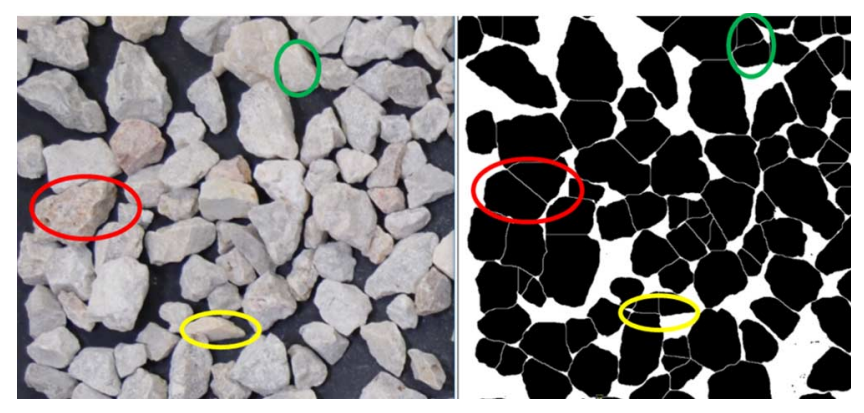

FIG. 5

Summary of the steps for image analysis through ImageJ.

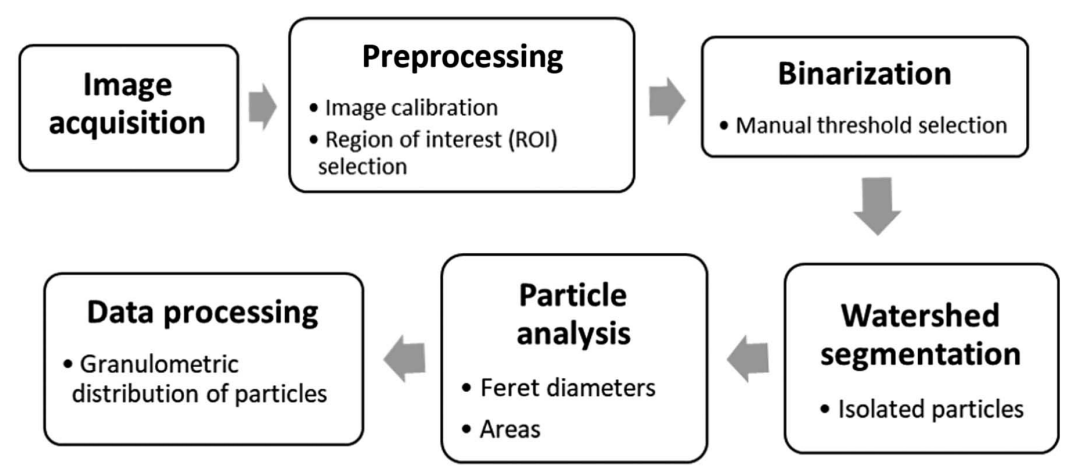




$$
\% \text { ret }=\frac{A^{\star} F_{\text {min }}}{\sum \text { Area }^{\star} F_{\text {min }}} x 100
$$

Taking the granulometry obtained by sieving as a reference, the errors in the determination of the granulometry through image analysis can be found. As the sieving procedure classifies the particles into larger bins than image analysis, to compare both results, it is necessary to obtain the retained percentage for those intermediate values in the case of sieving. This process was done by linear interpolation between the interval limits. With the retained percentage obtained by sieving $(D s)$ and the corresponding percentage for that size obtained through image analysis $(D i)$, we define the size error $(e)$ as follows:

$$
e=\frac{D_{s}}{D_{i}}
$$

\section{Results and Discussion}

Granulometries obtained by sieving and image analysis are presented in figure 6, wherein all results related to image processing are bonded to the equivalent diameter, as described in equation (1). The first assessment is that different illumination intensities did not produce relevant differences in the sizes obtained through image processing. However, image results systematically provided smaller size results than sieving in all cases, despite being parallel curves. To our understanding, this is due to the oversegmentation done by the watershed algorithms, since large stones can be divided into smaller pieces.

Results obtained from the cell phone camera show the same trend as those obtained through the Nikon camera, but in some cases, the oversegmentation shown by the iPhone is higher than that shown for the Nikon camera. This could be explained by the higher depth of field and the image enhancement methods that most cell phone cameras apply compared with the commercial camera. High depth of field and image postprocessing tend to reduce image blurring, providing sharp and highly contrasted pictures. Eventually, this effect may provide a rougher contour in the explored objects, thus giving the appearance of local concavities that increase the oversegmentation done by the watershed procedure.

These results are summarized and compared in Table 1, which shows the sizes corresponding to percentiles $60\left(D_{60}\right), 30\left(D_{30}\right)$, and $10\left(D_{10}\right)$, as well as the uniformity $\left(C_{u}\right)$ and curvature $\left(C_{c}\right)$ coefficients, as defined in Lambe and Whitman (2004). To better compare the obtained results, we have defined a correction factor (CF) that allows for modifying the results obtained through the image into the expected sieving ones. That factor was defined as the mean value of the error for all particles analyzed $(n)$ :

$$
C F=\frac{1}{n} \sum_{i=1}^{n} e_{i}
$$

Furthermore, as light intensity did not have any influence on the results, the mean value of all intensity results for the Nikon camera is used in Table 1. According to these results and following the Unified Soil Classification System, all samples are classified as poorly graded gravel.

Once knowing the particle sizes (both for sieving and image processing), we can obtain the particle size in px for all samples. These data are relevant because they link the image resolution to particle size in the samples to analyze. Of course, there will be a continuous progression from the smallest particle to the biggest one, following the lines given in figure 6 , but the ends of those intervals could provide guidance for the next related works. That is why the biggest and smallest particle sizes are described in px and $\mathrm{mm}$ for each analyzed sample in Table 2.

Analyzing the results shown in figure 6, it is clear that all curves obtained by image processing are displaced to the right with respect to the corresponding values obtained by sieving. This means that sizes obtained through sieving are higher than those obtained by image analysis. This characteristic can also be seen in the correction factor (following equation (4)) shown in Table 1. 
FIG. 6 Granulometries obtained by sieving and image analysis for Samples $(A) 1,(B) 2,(C)$ 3, (D) 4, (E) 5, and $(F) 6$.

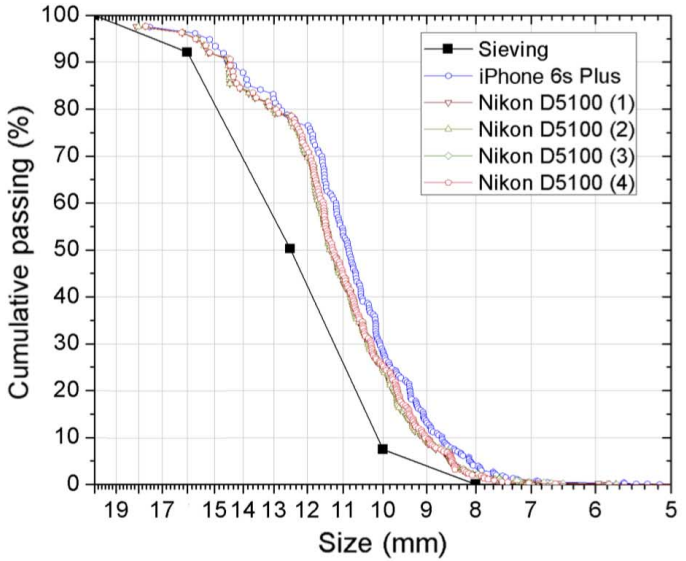

(A)

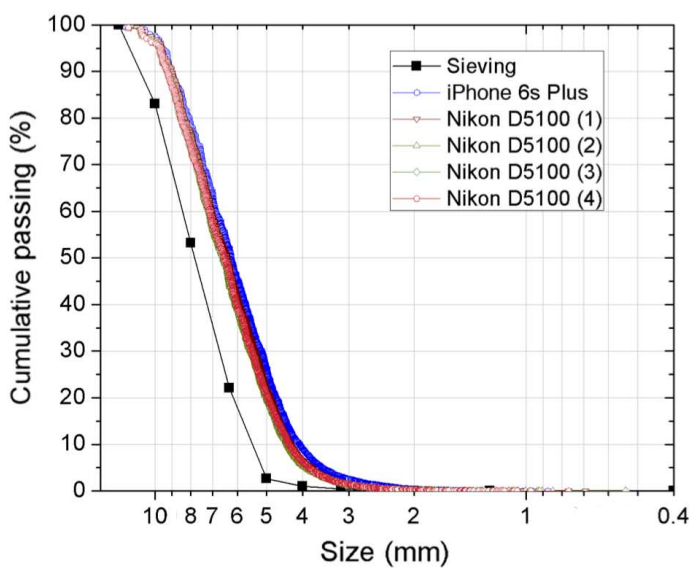

$(C)$

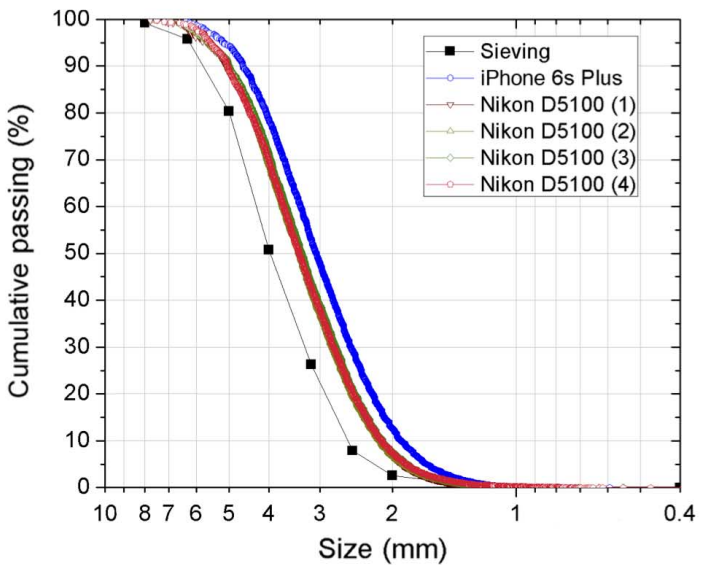

$(E)$

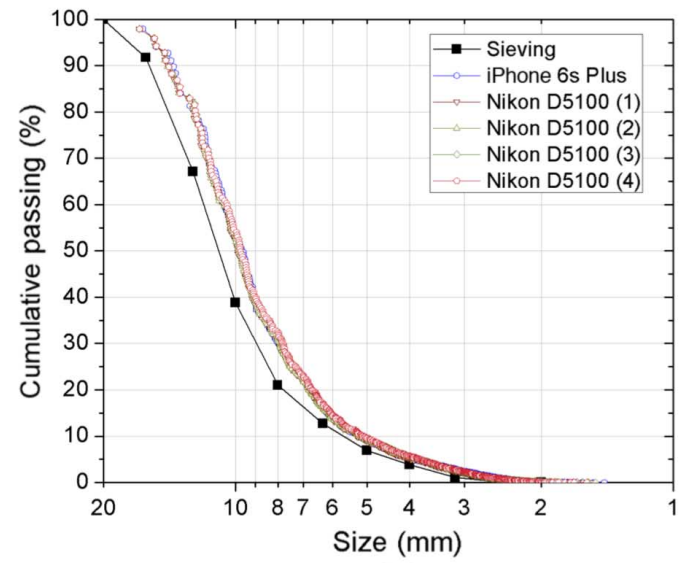

$(B)$

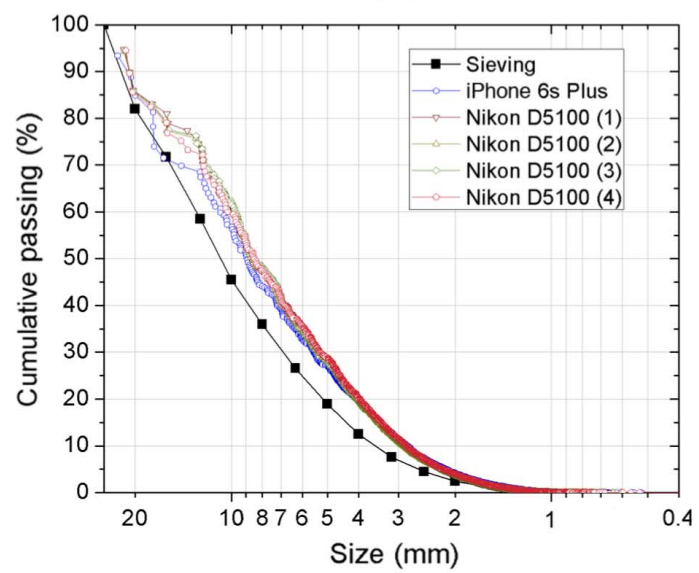

(D)

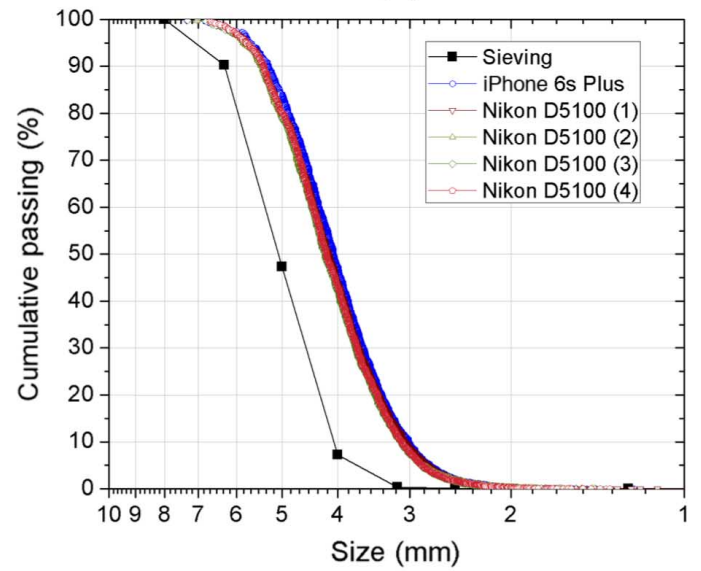

$(F)$

As we explained previously, the reason for this bias may be oversegmentation introduced by the watershed operation. Different techniques can be proposed to minimize this effect. One technique would consist of taking several pictures with the same samples after shaking the tray, thus changing the position of the stones and offering 
TABLE 1

Summary of results: granulometric parameters measured by sieving and image processing

\begin{tabular}{|c|c|c|c|c|c|c|c|c|}
\hline Sample & Granulometry & Method & $D_{60}, \mathrm{~mm}$ & $D_{30}, \mathrm{~mm}$ & $D_{10}, \mathrm{~mm}$ & $C_{u}$ & $C_{c}$ & Correction Factor \\
\hline \multirow[t]{3}{*}{ M1 } & Sieving & & 13.24 & 11.25 & 10.13 & 1.31 & 0.94 & $\ldots$ \\
\hline & Image analysis & iPhone 6s Plus & 11.20 & 10.08 & 8.68 & 1.29 & 1.05 & 1.15 \\
\hline & & Nikon D5100 & 11.62 & 10.33 & 9.04 & 1.29 & 1.02 & 1.12 \\
\hline \multirow[t]{3}{*}{ M2 } & Sieving & & 11.82 & 8.96 & 5.66 & 2.09 & 1.20 & $\ldots$ \\
\hline & Image analysis & iPhone 6s Plus & 10.59 & 7.99 & 5.18 & 2.04 & 1.16 & 1.18 \\
\hline & & Nikon D5100 & 10.58 & 7.87 & 5.19 & 2.04 & 1.13 & 1.15 \\
\hline \multirow[t]{3}{*}{ M3 } & Sieving & & 8.42 & 6.69 & 5.46 & 1.54 & 0.98 & $\cdots$ \\
\hline & Image analysis & iPhone 6s Plus & 6.86 & 5.16 & 4.07 & 1.69 & 0.95 & 1.35 \\
\hline & & Nikon D5100 & 7.13 & 5.48 & 4.37 & 1.63 & 0.97 & 1.27 \\
\hline \multirow[t]{3}{*}{ M4 } & Sieving & & 12.86 & 6.88 & 3.54 & 3.63 & 1.04 & $\ldots$ \\
\hline & Image analysis & iPhone 6s Plus & 10.83 & 5.52 & 2.82 & 3.84 & 1.00 & 1.22 \\
\hline & & Nikon D5100 & 9.87 & 5.35 & 2.87 & 3.44 & 1.01 & 1.19 \\
\hline \multirow[t]{3}{*}{ M5 } & Sieving & & 4.29 & 3.27 & 2.57 & 1.67 & 0.97 & $\ldots$ \\
\hline & Image analysis & iPhone 6s Plus & 3.35 & 2.53 & 1.91 & 1.76 & 1.00 & 1.30 \\
\hline & & Nikon D5100 & 3.68 & 2.79 & 2.13 & 1.72 & 0.99 & 1.16 \\
\hline \multirow[t]{3}{*}{ M6 } & Sieving & & 5.35 & 4.54 & 4.06 & 1.32 & 0.95 & $\cdots$ \\
\hline & Image analysis & iPhone 6s Plus & 4.31 & 3.61 & 3.01 & 1.43 & 1.00 & 1.29 \\
\hline & & Nikon D5100 & 4.42 & 3.71 & 3.09 & 1.43 & 1.01 & 1.26 \\
\hline
\end{tabular}

TABLE 2

Higher and smallest particle sizes in px for all samples (sizes in mm from fig. 6)

\begin{tabular}{lccccc}
\hline & \multicolumn{2}{c}{ Largest Particle Size } & & \multicolumn{2}{c}{ Smallest Particle Size } \\
\cline { 2 - 3 } \cline { 5 - 6 } Sample & $\mathrm{px}$ & $\mathrm{mm}$ & & $\mathrm{px}$ & $\mathrm{mm}$ \\
\hline M1 & 178 & 19.5 & & 64 & 7 \\
M2 & 184 & 20 & 18 & 2 \\
M3 & 110 & 12 & 18 & 2 \\
M4 & 187 & 20 & 9 & 1 \\
M5 & 71 & 8 & 9 & 1 \\
M6 & 71 & 8 & 27 & 3 \\
\hline
\end{tabular}

a different perspective. The granulometry curve will be then obtained by averaging the curves obtained for each different picture of the same sample. A more sophisticated technique would be changing the illumination point and again taking different pictures of the same sample. Image processing can also be used in combination with the previous explained method. It is known that blurring the sample softens the contours, thus avoiding some of the oversegmentations. One can also apply mathematical morphology to isolate the samples through an opening filter. Notice that all the methods proposed may avoid some oversegmentations, but not all of them, and they may also introduce segmentations in stones that were well characterized, so a statistical analysis of the introduced gain/loss is also necessary. In any case, detailed discussion of the oversegmentation minimization is, in our opinion, out of the scope of the manuscript since it is more related to image-processing techniques than to geotechnics.

Another possible explanation for this bias could be the lack of tridimensional information about the samples. In the images used, we can only see a planar view of the samples, ignoring the total volume. The lack of dimension can be relevant for the samples passing (or not) through the sieve aperture, thus distorting the granulometry statistics. The way to avoid this problem is to measure or infer the third dimension. Several ways have been described previously to do that in imaging. Some of them use stereography (Zheng and Hryciw 2017), structured 
light (Sun et al. 2019), or even tomography (Druckrey, Alshibli, and Al-Raoush 2016) to measure the third dimension. However, all of these methods involve more complex procedures than those included in the error evaluation that is done in this work.

Let us recall that the aim of this paper is to evaluate the errors obtained by using a simple procedure that can be described in only six steps (fig. 5) and that can be processed by using free software and some additional simple calculations. If errors are acceptable for some applications, or as a first result, this procedure could be used by anyone in any location since a commercial camera or a smartphone together with a laptop are more portable and conventional than a normalized set of sieves.

Differences between results obtained from commercial and smartphone cameras can be seen in the range of values for the correction factor, which is slighter higher for images taken by the iPhone (1.15-1.30) than those obtained by the Nikon camera (1.12-1.27). In any case, the amplitude range is very narrow in both cases, which gives the possibility of taking 1.23 and 1.19 as correction factors for the mobile and commercial cameras, respectively. Those values, obtained for each sample, are the mean of the correction factor obtained for each particle (equation (4)). In figure 7, we have plotted the variations of this parameter with respect to particle size in each sample. The measurement error is also included in the right axis for reference. Results from the smartphone camera show the highest errors, as expected by the results analyzed until this point, with the exception of Sample 4 for sizes higher than $3 \mathrm{~mm}$. In general, differences between errors from commercial and smartphone cameras depend on the particle size for each sample. For Sample 5, these differences are higher than for the rest of the samples, and they are maintained for almost all particle sizes. Sample 5 is one whose rounded particles, with particle sizes smaller than Samples 1 and 2 but similar to Sample 6, have very good agreement among the results from both cameras. To our knowledge, the only difference that could explain this behavior is the overlapping between particles in this sample, which, as can be seen in figure 8, is a bit more prevalent in

FIG. 7 Correction factor and error obtained for Samples $(A) 1,(B) 2,(C)$ 3, (D) 4, (E) 5, and (F) 6.

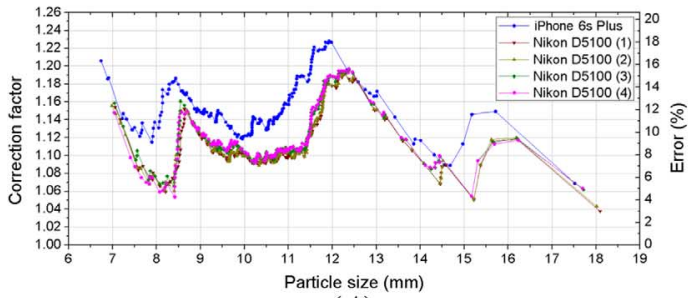

(A)

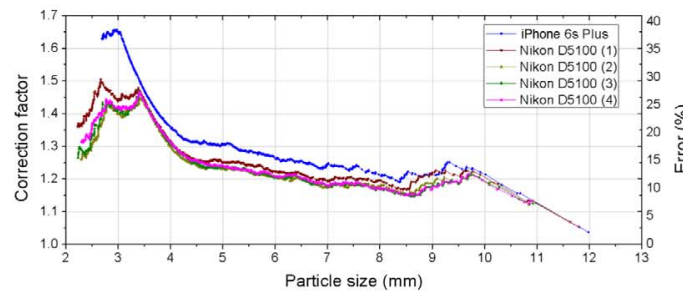

(C)

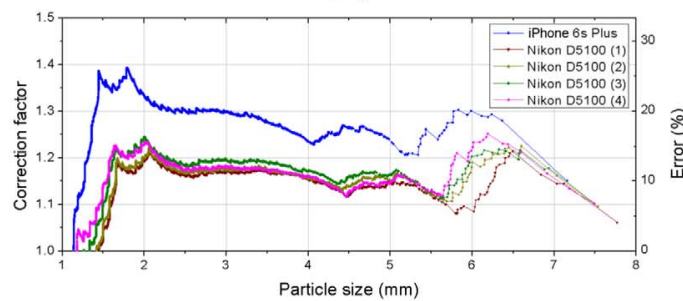

(E)

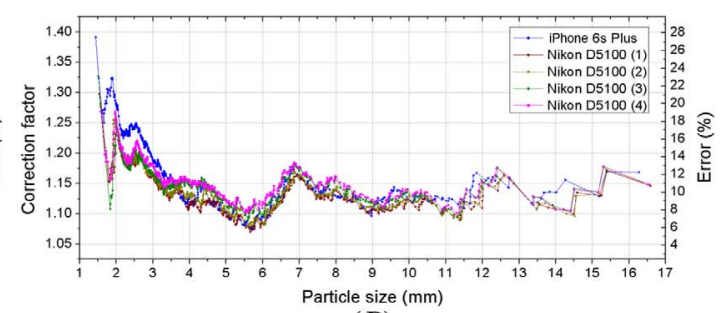

(B)

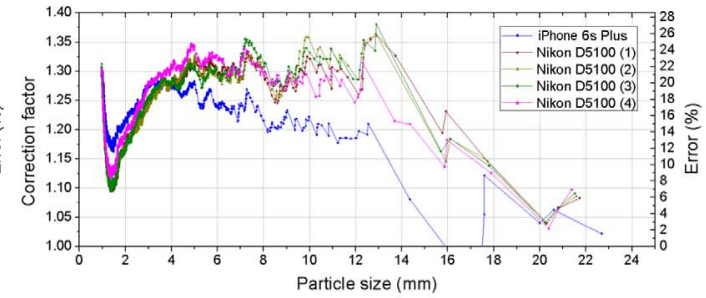

(D)

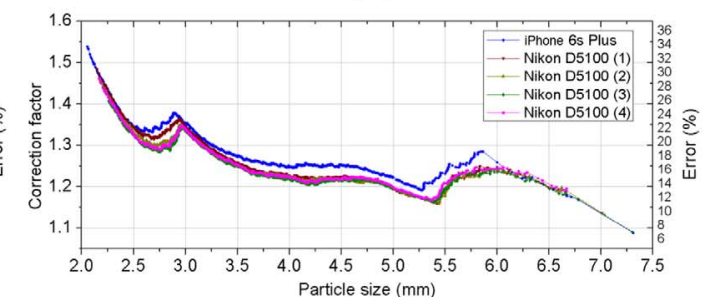

(F) 
FIG. 8

Difference in particles overlapping between Samples 5 (left) and 6 (right).
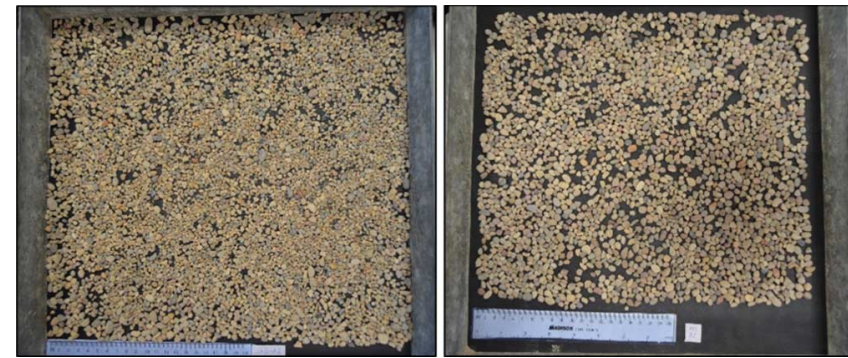

Sample 5 than Sample 6. This reinforces the importance of having a correct particle spread before taking the pictures.

To summarize the results from figure 7, mean and standard deviation (SD) values for the correction factor and the error are presented in Table 3. The SD for the correction factor is between 0.03 and 0.12 for the smartphone and 0.03 and 0.08 for the commercial camera. That means that the use of a unique correction factor for the whole sample will lead to small errors. The SD is only higher than 0.1 for the smartphone camera and Sample 3; for this case, the error reaches $25 \%$, the highest error found. However, from figure 7, one can see that those big errors came from the smallest part of the sample, i.e., particles below $3.5 \mathrm{~mm}$ that have a small representation in the whole Sample 3, as can be seen in figure 6. For the commercial camera, the highest errors are found for Samples 3 and 6, which show a more stepped granulometry (more uniformity in size) together with a small particle size (Table 1). This means that for granular materials such as sands, the method will have the highest errors, while for well-graded materials, the method will have the best results.

Regarding the errors obtained in the rest of the parameters that define the granulometry, they are summarized in Table 4 for results from the smartphone and Table 5 for results from the commercial camera. In all cases, errors from the smartphone camera are higher than those obtained from the commercial camera, as expected. Only in the case of the uniformity factor, which is the ratio between $D_{60}$ and $D_{10}$, the overmeasurement of both values is partially canceled, and as a result, the uniformity factor is not always worse for the smartphone than for the commercial camera.

\section{TABLE 3}

Mean values and SD of values shown in figure 7

\begin{tabular}{|c|c|c|c|c|c|}
\hline \multirow[b]{2}{*}{ Sample } & \multirow[b]{2}{*}{ Value } & \multicolumn{2}{|c|}{ Smartphone } & \multicolumn{2}{|c|}{ Commercial Camera (Mean Value for Cases 1-4) } \\
\hline & & Correction Factor & Error, \% & Correction Factor & Error, \% \\
\hline \multirow[t]{2}{*}{ M1 } & Mean & 1.15 & 13.28 & 1.12 & 10.38 \\
\hline & SD & 0.0297 & 2.20 & 0.0314 & 2.47 \\
\hline \multirow[t]{2}{*}{ M2 } & Mean & 1.18 & 14.75 & 1.15 & 13.20 \\
\hline & SD & 0.0592 & 4.22 & 0.0340 & 2.53 \\
\hline \multirow[t]{2}{*}{ M3 } & Mean & 1.35 & 25.34 & 1.27 & 20.91 \\
\hline & SD & 0.1213 & 6.08 & 0.0822 & 4.84 \\
\hline \multirow[t]{2}{*}{ M4 } & Mean & 1.22 & 18.03 & 1.19 & 15.79 \\
\hline & SD & 0.0375 & 2.53 & 0.0654 & 4.55 \\
\hline \multirow[t]{2}{*}{ M5 } & Mean & 1.30 & 22.55 & 1.16 & 13.85 \\
\hline & $\mathrm{SD}$ & 0.0744 & 5.26 & 0.0577 & 4.93 \\
\hline \multirow[t]{2}{*}{ M6 } & Mean & 1.29 & 22.46 & 1.26 & 20.44 \\
\hline & SD & 0.0561 & 3.23 & 0.0524 & 3.21 \\
\hline
\end{tabular}

Note: Values for correction factors given in Table 1 are in bold. 
TABLE 4

Errors obtained in granulometric parameters measured by image processing using a smartphone

\begin{tabular}{|c|c|c|c|c|c|c|c|}
\hline Errors for Smartphone, \% & & & $D_{60}$ & $D_{30}$ & $D_{10}$ & $C_{u}$ & $C_{c}$ \\
\hline \multirow[t]{6}{*}{ Rounded Particles } & M1 & & 15.45 & 10.37 & 14.32 & 1.32 & 10.89 \\
\hline & M2 & & 10.45 & 10.82 & 8.49 & 2.14 & 2.95 \\
\hline & M5 & & 21.94 & 22.68 & 25.74 & 5.12 & 3.12 \\
\hline & M6 & & 19.40 & 20.46 & 25.82 & 8.66 & 5.82 \\
\hline & & Mean & 16.81 & 16.08 & 18.59 & 4.31 & 5.69 \\
\hline & & SD & 5.01 & 6.40 & 8.63 & 3.33 & 3.70 \\
\hline \multirow[t]{4}{*}{ Sharp Particles } & M3 & & 18.44 & 22.96 & 25.37 & 9.29 & 2.48 \\
\hline & M4 & & 15.84 & 19.70 & 20.52 & 5.89 & 3.59 \\
\hline & & Mean & 17.14 & 21.33 & 22.94 & 7.59 & 3.03 \\
\hline & & $\mathrm{SD}$ & 1.84 & 2.31 & 3.43 & 2.40 & 0.78 \\
\hline
\end{tabular}

TABLE 5

Errors obtained in granulometric parameters measured by image processing using a commercial camera

\begin{tabular}{|c|c|c|c|c|c|c|c|}
\hline Errors for Commercial Camera, \% & & & $D_{60}$ & $D_{30}$ & $D_{10}$ & $C_{u}$ & $C_{c}$ \\
\hline \multirow[t]{6}{*}{ Rounded Particles } & M1 & & 12.28 & 8.16 & 10.80 & 1.66 & 7.78 \\
\hline & M2 & & 10.52 & 12.16 & 8.36 & 2.34 & 5.90 \\
\hline & M5 & & 14.19 & 14.75 & 16.87 & 3.23 & 1.88 \\
\hline & M6 & & 17.41 & 18.38 & 23.92 & 8.55 & 6.02 \\
\hline & & Mean & 13.6 & 13.36 & 14.99 & 3.95 & 5.39 \\
\hline & & SD & 2.95 & 4.31 & 6.95 & 3.14 & 2.50 \\
\hline \multirow[t]{4}{*}{ Sharp Particles } & M3 & & 15.32 & 18.11 & 19.96 & 5.81 & 1.06 \\
\hline & M4 & & 23.28 & 22.27 & 19.08 & 5.17 & 2.78 \\
\hline & & Mean & 19.3 & 20.19 & 19.52 & 5.49 & 1.92 \\
\hline & & $\mathrm{SD}$ & 5.62 & 2.94 & 0.62 & 0.45 & 1.22 \\
\hline
\end{tabular}

Results from samples with sharp particles are slightly worse than those obtained from samples with rounded particles. Errors in the determination of percentiles are almost always below $20 \%$, but errors in the uniformity and curvature coefficients are much smaller, below $8 \%$.

\section{Conclusions}

In this paper, the errors that have arisen from a straightforward image process to obtain particle granulometries are analyzed. Different intensity lights were used as well as two different common devices to obtain the images: a commercial camera and a smartphone. The errors were found by comparison with sieve results, which is taken as the gold standard method despite its issues in the determination of particle sizes. From the results and discussion, we can conclude the following:

- The obtained granulometric curves by image processing are parallel to those obtained by sieving. The image procedure described here gives smaller particles than sieving.

- The influence of different light intensities is insignificant in the errors obtained. The SD obtained for the relative error for all samples is between 0.31 and $0.78 \%$.

- Results from the smartphone camera were slightly worse than those obtained from the commercial camera. Our hypothesis is that smartphone cameras may implement some image-processing algorithms to improve the final results, thus affecting the sharpness and color of the image. This may affect the contour detection and introduce a small bias in the particle classification.

- Image sizing can be approximated to sieving results using the same factor for all sizes in the sample because the $\mathrm{SD}$ of that factor throughout the whole sample is smaller than $10 \%$ of the mean correction factor. 
- The range for the correction factors we found for all samples is very narrow: from 1.12 to 1.27 for the commercial camera and from 1.15 to 1.30 for the smartphone. That narrow interval means that by simply using the mean value as the correction factor, the error will not increase extremely. Additionally, it means that the value obtained here for the correction factor will be robust for other experiments.

- The recommended values for the correction factor are 1.19 for commercial cameras and 1.23 for smartphone cameras.

- Without using the correction factor, the errors obtained a range from 10 to $20 \%$ for commercial cameras and from 13 to $25 \%$ for smartphone cameras. Once the correction factor is applied, the maximum error found is $5 \%$ for all samples.

- Curvature and uniformity coefficients obtained by image processing have an error between 1.06 and $10.89 \%$, but for a part of the sample that is smaller than the rest, that range increases up to 8.16 and $25.82 \%$.

- For samples with rounded particles, the method is slightly more accurate than for sharp particle samples. However, the arrangement of the sample in the tray can change the accuracy of the method. Contact between particles can happen, but the base of the tray should be as visible as possible. That implies that the sample quantity should be small enough to fit in the image with the recommended spread, and this leads to possible problems of inhomogeneities in the sample partition.

In summary, the method analyzed in this paper has an average error of $15 \%$, which is accurate enough for many applications, taking into account that the resources and knowledge needed is very little. Using a robust coefficient factor of around 1.2, those errors drop down to $5 \%$, which could be very close to the errors that sieving presents. Therefore, only using a smartphone camera, free software, and a laptop (without any additional light), an acceptable particle sizing analysis of a sample can be done with accuracy similar to sieving, which enables the possibility of in situ experiments without complex setups.

\section{References}

Abbireddy, C. O. R. and C. R. I. Clayton. 2009. “A Review of Modern Particle Sizing Methods.” Proceedings of the Institution of Civil Engineers - Geotechnical Engineering 162, no. 4 (August): 193-201. https://doi.org/10.1680/geng.2009.162.4.193

Altuhafi, F., C. O'Sullivan, and I. Cavarretta. 2013. "Analysis of an Image-Based Method to Quantify the Size and Shape of Sand Particles." Journal of Geotechnical \& Geoenvironmental Engineering 139, no. 8 (August): 1290-1307. https://doi.org/10. 1061/(ASCE)GT.1943-5606.0000855

Annegarn, H. J., H. Storms, R. E. van Grieken, and P. A. Booth-Jones. 1987. “Composition and Size of Individual Particles from a Gold Mine Atmosphere.” Mining Science and Technology 5, no. 2 (July): 111-119. https://doi.org/10.1016/S0167-9031(87) 90345-8

Asociación Española de Normalización. Tests for Geometrical Properties of Aggregates - Part 1: Determination of Particle Size Distribution - Sieving Method. UNE-EN 933-1:2012. Madrid, Spain: Asociación Española de Normalización, approved July 11, 2012.

Barksdale, R. D., M. A. Kemp, W. J. Sheffield, and J. L. Hubbard. 1991. "Measurement of Aggregate Shape, Surface Area, and Roughness." Transportation Research Record 1301: 107-116.

Buscombe, D., D. M. Rubin, and J. A. Warrick. 2010. "A Universal Approximation of Grain Size from Images of Noncohesive Sediment.” Journal of Geophysical Research: Earth Surface 115, no. F2 (June): F02015. https://doi.org/10.1029/2009JF001477

Butler, J. B., S. N. Lane, and J. H. Chandler. 2001. "Automated Extraction of Grain-Size Data from Gravel Surfaces Using Digital Image Processing.” Journal of Hydraulic Research 39, no. 5: 519-529. https://doi.org/10.1080/00221686.2001.9628276

Cavarretta, I., M. Coop, and C. O'Sullivan. 2010. “The Influence of Particle Characteristics on the Behaviour of Coarse Grained Soils." Géotechnique 60, no. 6 (June): 413-423. https://doi.org/10.1680/geot.2010.60.6.413

Chávez, G. M., D. Sarocchi, E. A. Santana, and L. Borselli. 2015. "Optical Granulometric Analysis of Sedimentary Deposits by Color Segmentation-Based Software: OPTGRAN-CS.” Computers and Geosciences 85 (December): 248-257. https://doi. org/10.1016/j.cageo.2015.09.007

Corvari, V., L. O. Narhi, T. M. Spitznagel, N. Afonina, S. Cao, P. Cash, I. Cecchini, et al. 2015. “Subvisible (2-100 $\mu$ m) Particle Analysis during Biotherapeutic Drug Product Development: Part 2, Experience with the Application of Subvisible Particle Analysis." Biologicals 43, no. 6 (November): 457-473. https://doi.org/10.1016/j.biologicals.2015.07.011

Dipova, N. 2017. "Determining the Grain Size Distribution of Granular Soils Using Image Analysis." Acta Geotechnica Slovenica 14: 29-37.

Druckrey, A. M., K. A. Alshibli, and R. I. Al-Raoush. 2016. "3D Characterization of Sand Particle-to-Particle Contact and Morphology 2016.” Computers and Geotechnics 74 (April): 26-35. https://doi.org/10.1016/j.compgeo.2015.12.014 
Fernlund, J. M. R. 1998. “The Effect of Particle Form on Sieve Analysis: A Test by Image Analysis.” Engineering Geology 50, nos. 1-2 (September): 111-124. https://doi.org/10.1016/S0013-7952(98)00004-0

Fernlund, J. M. R., R. W. Zimmerman, and D. Kragic. 2007. "Influence of Volume/Mass on Grain-Size Curves and Conversion of Image-Analysis Size to Sieve Size.” Engineering Geology 90, nos. 3-4 (March): 124-137. https://doi.org/10.1016/j.enggeo. 2006.12.007

Ghalib, A. M. and R. D. Hryciw. 1999. "Soil Particle Size Distribution by Mosaic Imaging and Watershed Analysis." Journal of Computing in Civil Engineering 13, no. 2 (April): 80-87. https://doi.org/10.1061/(ASCE)0887-3801(1999)13:2(80)

Gonzalez, R. C. and R. E. Woods. 2008. Digital Image Processing. Upper Saddle River, NJ: Prentice Hall.

Graham, D. J., I. Reid, and S. P. Rice. 2005. “Automated Sizing of Coarse-Grained Sediments: Image-Processing Procedures.” Mathematical Geology 37, no. 1 (January): 1-28. https://doi.org/10.1007/s11004-005-8745-x

Hazen, A. 1892. "Some Physical Properties of Sands and Gravels, with Special Reference to Their Use in Filtration." In 24th Annual Report, 539-556. Boston, MA: Massachusetts State Board of Health.

Hazen, A. 1911. "Discussion of 'Dams on Sand Foundations' by A. C. Koenig." Transactions of the American Society of Civil Engineers 73: 199-203.

Kodikara, J., T. Islam, and A. Sounthararajah. 2018. "Review of Soil Compaction: History and Recent Developments." Transportation Geotechnics 17 (December): 24-34. https://doi.org/10.1016/j.trgeo.2018.09.006

Kumara, G. H. A. J. J., K. Hayano, and K. Ogiwara. 2012. "Image Analysis Techniques on Evaluation of Particle Size Distribution of Gravel." International Journal of GEOMATE 3, no. 1 (September): 290-297. https://doi.org/10.21660/ 2012.5.1261

Kuo, C.-Y., R. S. Rollings, and L. N. Lynch. 1998. “Morphological Study of Coarse Aggregates Using Image Analysis.” Journal of Materials in Civil Engineering 10, no. 3 (August): 135-142. https://doi.org/10.1061/(ASCE)0899-1561(1998)10:3(135)

Kwan, A. K. H., C. F. Mora, and H. C. Chan. 1999. "Particle Shape Analysis of Coarse Aggregate Using Digital Image Processing." Cement and Concrete Research 29, no. 9 (September): 1403-1410. https://doi.org/10.1016/S0008-8846(99) 00105-2

Lambe, T. W. and R. V. Whitman. 2004. Mecánica de suelos. Mexico City, Mexico: Limusa.

Li, W.-Y., S.-H. Yan, Y.-P. Yin, Y. Li, T.-B. Liang, F. Gu, Z.-M. Dai, and Z.-L. Wang. 2008. "Comparison of Starch Granule Size Distribution between Hard and Soft Wheat Cultivars in Eastern China.” Agricultural Sciences in China 7, no. 8 (August): 907-914. https://doi.org/10.1016/S1671-2927(08)60129-7

Marinelli, F., E. Falcieri, S. Squarzoni, R. Del Coco, N. Zini, L. Manzoli, and N. M. Maraldi. 1990. "Image Analysis of the Chromatin Organization in the Nuclear Domains of Freeze Fractured Hepatocytes and Lymphocytes." Biology of the Cell 70, no. 3: 107-119. https://doi.org/10.1016/0248-4900(90)90366-B

Mora, C. F. and A. K. H. Kwan. 2000. "Sphericity, Shape Factor, and Convexity Measurement of Coarse Aggregate for Concrete Using Digital Image Processing." Cement and Concrete Research 30, no. 3 (March): 351-358. https://doi.org/10.1016/S00088846(99)00259-8

Mora, C. F., A. K. H. Kwan, and H. C. Chan. 1998. "Particle Size Distribution Analysis of Coarse Aggregate Using Digital Image Processing.” Cement and Concrete Research 28, no. 6 (June): 921-932. https://doi.org/10.1016/S0008-8846(98)00043-X

Motte, J.-C., J.-Y. Delenne, C. Barron, É. Dubreucq, and C. Mayer-Laigle. 2017. "Elastic Properties of Packing of Granulated Cork: Effect of Particle Size.” Industrial Crops and Products 99 (May): 126-134. https://doi.org/10.1016/j.indcrop.2017.01. 043

Raschke, S. A. and R. D. Hryciw. 1997. “Grain-Size Distribution of Granular Soils by Computer Vision.” Geotechnical Testing Journal 20, no. 4 (December): 433-442. https://doi.org/10.1520/GTJ10410J

Sarocchi, D., L. Borselli, and J. L. Macías. 2005. "Construcción de perfiles granulométricos de depósitos piroclásticos por métodos ópticos." Revista mexicana de ciencias geológicas 22, no. 3: 371-382.

Sezgin M. and B. Sankur. 2004. "Survey over Image Thresholding Techniques and Quantitative Performance Evaluation." Journal of Electronic Imaging 13, no. 1 (January): 146-165. https://doi.org/10.1117/1.1631315

Sun, Q., Y. Zheng, B. Li, J. Zheng, and Z. Wang. 2019. “Three-Dimensional Particle Size and Shaper Characterisation Using Structural Light.” Géotechnique Letters 9, no. 1 (March): 72-78. https://doi.org/10.1680/jgele.18.00207

Wikipedia. 2020. “Otsu's Method.” Wikipedia. http://web.archive.org/web/20200330133418/https://en.wikipedia.org/wiki/ Otsu\%27s_method

Xiao, Y. X., C. F. Lee, and S. J. Wang. 2002. "Particle-Size Distribution of Interlayer Shear Zone Material and Its Implications in Geological Processes-A Case Study in China.” Engineering Geology 66, nos. 3-4 (November): 221-232. https://doi.org/10. 1016/S0013-7952(02)00043-1

Zheng, J. and R. D. Hryciw. 2017. "Soil Particle Size and Shape Distributions by Stereophotography and Image Analysis." Geotechnical Testing Journal 40, no. 2 (March): 317-328. https://doi.org/10.1520/GTJ20160165 